\title{
Lunar deflections of the vertical and their distribution
}

\author{
Jinyun Guo ${ }^{1,2^{\star}}$, Yu Sun ${ }^{1,2}$, Xiaotao Chang $^{2,3}$, Fanlin Yang ${ }^{1,2}$ \\ ${ }^{1}$ College of Geodesy and Geomatics, Shandong University of Science and Technology, Qingdao, China; ${ }^{*}$ Corresponding Author: \\ jinyunguo1@126.com \\ ${ }^{2}$ Key Laboratory of Surveying and Mapping Technology on Island and Reef of SBSM, Qingdao, China; \\ ${ }^{3}$ Satellite Surveying and Mapping Application Center of SBSM, Beijing, China.
}

Received 4 February 2011; revised 20 March 2011; accepted 6 April 2011.

\begin{abstract}
The deflection of the vertical reflects the mass distribution and density anomaly of celestial bodies. Lunar deflections of the vertical include directional information of the Moon's gravity field. SGM90d, recovered from SELENE mission, revealed the lunar far side gravity field for the first time in history owes to 4-way Doppler data. Lunar deflections of the vertical and their meridional and prime vertical components are calculated from SGM90d, and then their global distributions are also given in the paper. The gradients of lunar deflections of the vertical are defined and computed as well. The correlations between the lunar deflections of the vertical and the lunar terrain have been fully discussed. Many different characteristics of lunar deflections of vertical have been found between the near side and the far side of the Moon, which may be caused from the lithospheric compensation and the uplifting of mantle.
\end{abstract}

Keywords: Lunar Deflections of the Vertical; Lunar Gravity Field; Lunar Topography; Gradient of Vertical Deflection

\section{INTRODUCTION}

The Moon is the natural celestial body closest to our living planet, Earth. There are many fantasies about the Moon since it could be the second home for human. Many lunar probing missions had been implemented by the Soviet Union, the United States, Europe, Japan, China and India since 1960s. Depending on mission objectives, lunar spacecrafts were either used to detect moon on a certain orbit or impacted on the surface of the Moon at last or soft landed on it with or without astronauts. The primary science objectives of lunar exploration including determination of the structure of the lunar interior, from crust to core and further understanding of the thermal evolution of the Moon [1]. Lunar gravity field, which contains abundant information related to lunar internal mass distribution, plays a key role in orbit determination and controlling lunar spacecrafts.

Abundant lunar gravity information can be extracted from the ground tracking data of lunar satellite and observations from lunar explorers, which are scientific data used to refine the lunar gravity field model [2]. Study of lunar gravity with ground tracking data began with the Soviet Union spacecraft Luna 10 lunched in 1966. Many lunar probing missions have been implemented since then. Lunar Orbiter (LO-I, II, III, IV, and V), mini satellites of Apollo 15 and 16 (A15 and A16ss), Clementine, Lunar Prospector (LP), SMART-1, SELENE, ChangE and Chandrayaan provided plenty ground tracking data to recovery lunar gravity field models. Observables from these lunar missions include ranges, range rates, two-/three-/four-way Doppler observations, D-VLBI data, direction data and craft-borne laser altimetric terrain data. These data include more frequences of lunar gravity field information [3-8]. Existing high-degree lunar gravity field models, such as LP100J, LP100K and LP165P, were estimated from tracking data of LP and historical lunar crafts before SELENE mission was implemented $[5,9,10]$. It is a known fact that there are no direct observations of the farside, and the observing precision at the Moon edge is low. Meantime the distribution of tracking data is not even and there are more data at the lunar equatorial area. So these lunar gravity models have much higher resolution at the nearside and lower resolution at the farside. Also, there are more errors and aliasing at the truncated degrees for these models [5]. SELENE can directly be tracked at the nearside and the farside from the earth stations, and the two-/four-way Doppler data and ranges can be collected $[11,12]$. A new lunar gravity field model SGM90d to degree and order of 90 has been developed from the SELENE-tracking data and historical data [13]. SGM90d is the first model including the direct observations at the farside. The lunar 
gravity field model is mainly used to study the lunar air-free gravity anomalies and the Bouguer anomalies, and discuss the lunar gravity anomalies at mascons and basins.

The deflection of the vertical is an angle between the direction of gravity and a reference direction [14]. Lunar deflections of the vertical indicate the slope of selenoid relative to the reference lunar ellipsoid, as well as the angle between the practical lunar vertical and the normal lunar gravity direction. So the lunar deflections of the vertical include information of internal lunar mass distribution and anomaly. Lunar gravity vector is composed of the vertical deflection and gravity anomaly based on a reference normal gravity. Lunar deflection of the vertical, as one basic observation in the lunar geophysics and selenodesy, can provide abundant information of lunar gravity field and selenoid. So the lunar deflections of the vertical are very important for the study of lunar gravity field. But we cannot directly precisely measure the vertical deflections on the lunar surface at present. Lunar deflections of the vertical and its distribution are calculated based on SGM90d in this paper. Then the correlation between lunar deflections of the vertical and lunar topography is analyzed. Differences of vertical deflections on lunar mascons and basins are also discussed.

\section{LUNAR DEFLECTIONS OF THE VERTICAL}

According to the definition of Molodensky deflection of the vertical [15], lunar deflection of the vertical can be calculated based on the lunar gravity model from the following formulas:

$$
\begin{gathered}
\xi=-\frac{G M}{\gamma r^{2}} \sum_{n=2}^{N}\left(\frac{a}{r}\right)^{n} \\
\cdot \sum_{m=0}^{n}\left(\bar{C}_{n m} \cos m \lambda+\bar{S}_{n m} \sin m \lambda\right) \frac{\mathrm{d} \bar{P}_{n m}(\sin \varphi)}{\mathrm{d} \varphi} \\
\eta=-\frac{G M}{\gamma r^{2} \cos \varphi} \sum_{n=2}^{N}\left(\frac{a}{r}\right)^{n} \\
\cdot \sum_{m=0}^{n} m\left(-\bar{C}_{n m} \sin m \lambda+\bar{S}_{n m} \cos m \lambda\right) \bar{P}_{n m}(\sin \varphi) \\
V D=\sqrt{\xi^{2}+\eta^{2}} \\
\tan \alpha=\frac{\eta}{\xi}
\end{gathered}
$$

where $\xi$ and $\eta$ the meridional and the prime vertical components, respectively; $V D$ the deflection of the vertical; $G M$ the lunar gravitational constant; $\gamma$ the normal lunar gravity; $(r, \varphi, \lambda)$ the spherical coordinates, $N$ the highest degree of the model used; $n$ and $m$ degree and order of the gravity field model; $\bar{C}_{n m}$ and $\bar{S}_{n m}$ the fully normalized lunar potential coefficients; $\bar{P}_{n m}(\sin \varphi)$ the fully normalized associated Legendre function, and $\alpha$ the azimuth of the vertical deflection.

Namiki et al. have developed a new lunar gravity field model up to degree and order of 90 named SGM90d. Tracking data used to determine this gravity model includes SELENE tracking data from Oct. 31, 2007 to Apr. 1, 2008 as well as historical lunar satellites tracking data [13]. Though has a lower degree and order, SGM90d reveals lunar far side ring shaped gravity features in a much higher resolution than existing lunar gravity models like LP100k. This significant improvement owes to SELENE 4way Doppler observations, which enables direct tracking of the satellite over far side of the Moon. The deflection of vertical and its meridional and prime vertical components and direction are calculated from SGM90d, drew with GMT [16], shown in Figure 1.

The global distribution of meridional components of lunar vertical deflections has been shown in Figure 1(a). Impacted basins locating at the near side including Imbrium, Serentitatis, Crisium and Humorum have similar symmetrical patterns with positive meridional components at east and negative ones at west. Nectaris and Smythii have rather complicated distribution patterns, which are positive-negative-positive-negative (from west to east). Orientale, Hertzsprung, Freundlich-Sharonov, Moscoviense, Apollo and Mendel-Rydberg locating at the farside, on the other hand, have negative-positive-negative-positive (from west to east) distribution patterns.

The global distribution of prime vertical components presented in Figure 1(b) also shows similar symmetrical pattern, but with a different symmetry axis direction. Four impacted basins, Imbrium, Serenitatis, Crisium and Nectaris, have positive prime vertical components at northern part of the basins and negative ones on the other haft. Humorum, Orientale and Mendel-Rydberg have negative-positive-negative-positive distribution pat- terns in north-south direction.

From the global distribution of lunar deflections of the vertical (shown in Figure 1(c)), ring shaped distribution patterns can be fond at impacted basins including Imbrium, Serenitatis, Crisium, Nectaris and Humorum. Vertical deflections are very large at the periphery of the basins and much smaller in the center area. The circles formed by large deflections of the vertical closely related to the lunar terrain and clearly indicate the boundaries of original mascons [13]. The large deflections of the vertical at Orientale formed two concentric circles. The outer ring is by the edge of the basin and inner one seems to be the margin of gravity anomaly in the basin. And it is the same case for other basins like Hertzsprung, 


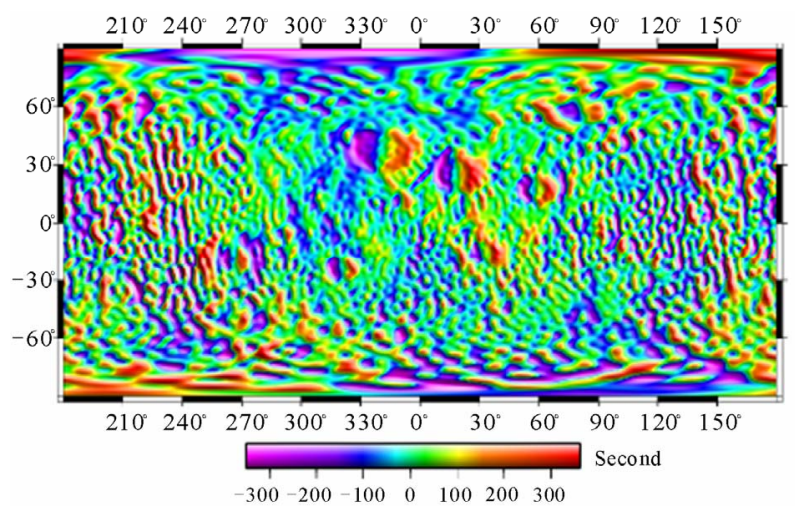

(a)

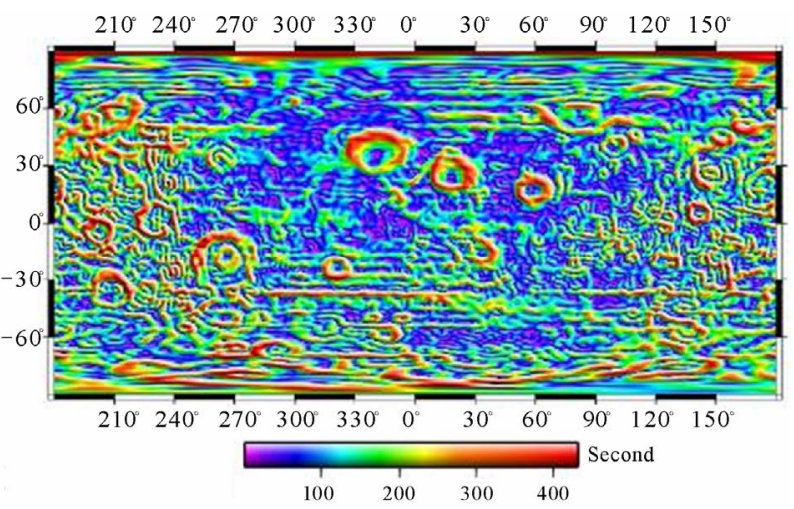

(c)

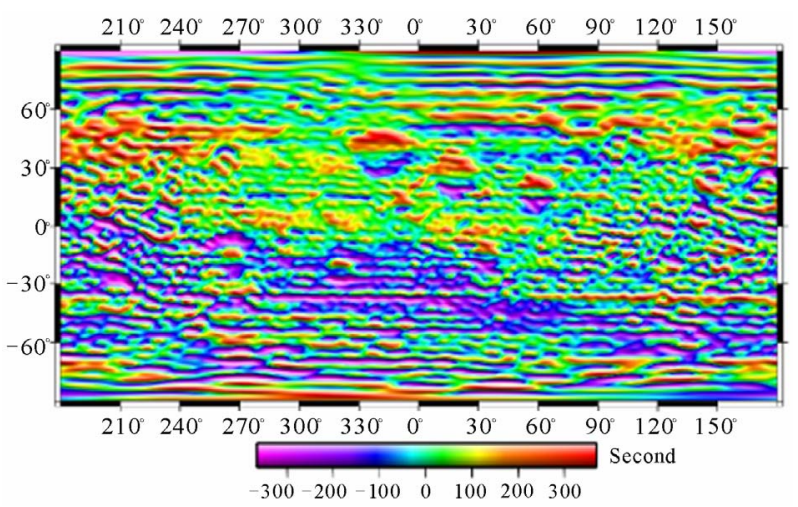

(b)

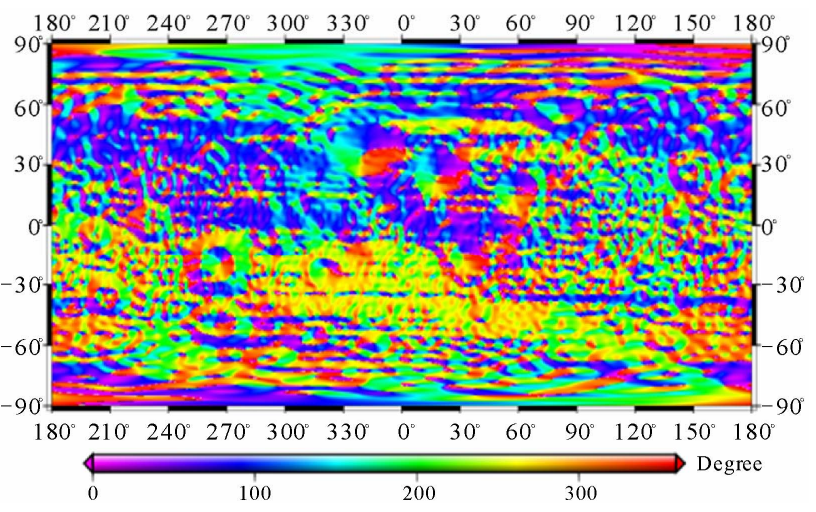

(d)

Figure 1. Distribution of lunar vertical deflections: (a) meridional, (b) prime vertical, (c) deflection of the vertical and (d) azimuth; the X-coordinate represents longitude and the Y-coordinate represents latitude.

Lorentz, Korolev, Apollo, Freundlich-Sharonov and Moscoviense. Lorentz and Korolev have same air-free gravity anomalies and Bouguer anomalies in the whole basin area. But for Orientale, Hertzsprung, Apollo, FreundlichSharonov and Moscoviense, the air-free gravity anomalies are 20\% - 60\% less than the Bouguer anomaly [13].

Presented in Figure 1(d) is the global distribution of azimuth of the vertical deflection. It can be seen that the azimuth changed counterclockwise from $0^{\circ}-360^{\circ}$ at several famous basins with single ring shaped vertical deflections. In the case of basins with double-ring vertical deflections, each of the two ring has same changes described above, but there's $180^{\circ}$ difference between the outer and inner rings.

\section{GRADIENTS OF LUNAR VERTICAL DEFLECTIONS}

The gradient of lunar vertical deflection is defined as

$$
s=\sqrt{s_{1}^{2}+s_{2}^{2}}
$$

where $s_{1}$ and $s_{2}$ are the numerical differentials of meridionla component and prime vertical component of vertical deflection to latitude and longitude, respectively.
So the gradient of lunar vertical deflection can be calculated with SGM90d, shown in Figure 2. We can find that Imbrium, Serenitatis, Crisium, Smythii, Nectaris and Humorum have gradients with the annulus distributions. The gradient distributions in other basins are very complicated.

\section{CORRELATIONS BETWEEN LUNAR DEFLECTIONS OF THE VERTICAL AND TERRAIN}

Lunar deflections of the vertical reflect abundant information of mass anomaly and its distribution, and closely correlate with the lunar terrain. With laser ranging data (Now. 272007 to Jan. 22 2008) from Chang'E, Ping et al. developed a lunar terrain model CLTM-s01 to degree and order 360 in the form of spherical expansion [17]. CLTM-s01 has better spatial coverage, precision $(31 \mathrm{~m})$ and resolution $\left(0.25^{\circ}\right)$ than any previous models. Lunar terrain and its gradient derived greater than 0.5 , from CLTM-s01 have been shown in Figures 3 and 4, respectively.

The following formula is used to calculate correlations between the vertical deflections and the terrain gradients at the nearside and farside, 


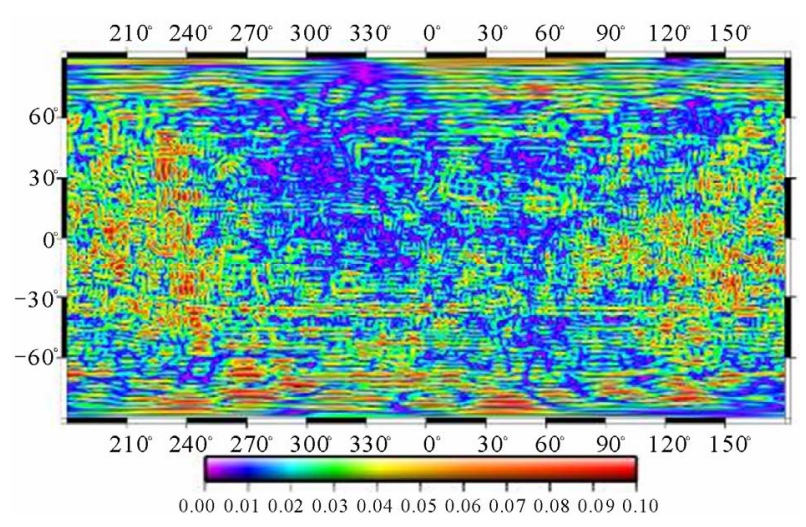

Figure 2. Gradients of luanr veritical deflections, the X-coordinate represents longitude and the Y-coordinate represents latitude.

$$
\text { Correlation }=\frac{\sum(D O V-\overline{D O V}) \cdot(T-\bar{T})}{\sqrt{\sum(D O V-\overline{D O V})^{2} \sum(T-\bar{T})^{2}}}
$$

where $D O V$ and $T$ are vertical deflections and elevations on the nearside or the farside of the Moon, respectively; $D O V$ and $T$ are the mean value of vertical deflections and the mean value of elevations on the nearside or the farside of the Moon, respectively.

Correlations between the vertical deflections and the terrain gradients at the nearside and the farside are 0.427 , and 0.416 respectively. From Figures 1(c) and 4, we can find that there are high correlations between the lunar terrain and the vertical deflections, which indicate that the vertical deflection can be caused from the mass anomaly in the lunar shell and surface to a large extent, and the internal mass in the Moon may be uniformly present. So the evolution in the internal Moon on the whole may end.

Lunar deflections of the vertical are calculated from SGM90d to degree and order 90, but CLTM-s01 is to degree and order 360 . Figure 5 shows the correlation between the geopotential coefficients and the terrain spherical harmonic coefficients to degree and order 90 . We can find that the correlations for degrees 30 to 80 are which indicate that the lunar terrain on this scale may largely contribute to the lunar gravity anomalies.

\section{CONCLUSIONS}

The lunar deflections of the vertical and their gradients are calculated from lunar gravity model SGM90d. Analyzing different distribution patterns of vertical deflections at several main basins shows that basins locate at the nearside of the Moon have different characteristics compared with those at the farside. The differences can be cause by the lithospheric compensation and the lunar

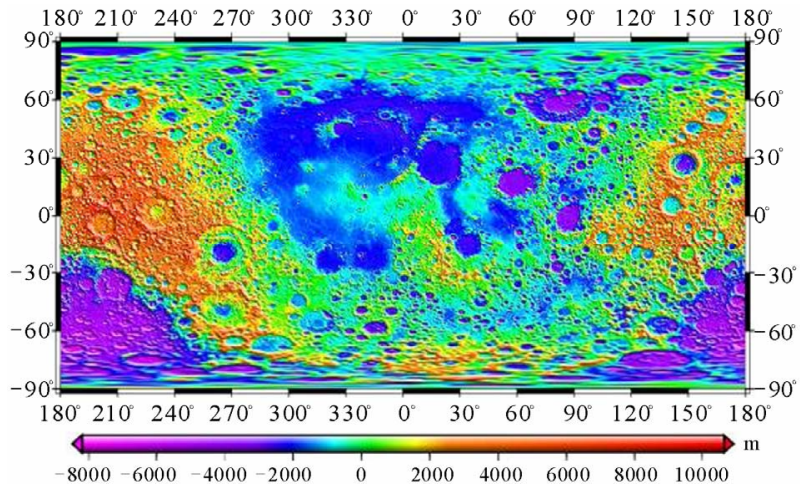

Figure 3. Lunar terrian derived from CLTM-s01, the X-coordinate represents longitude and the Y-coordinate represents latitude.

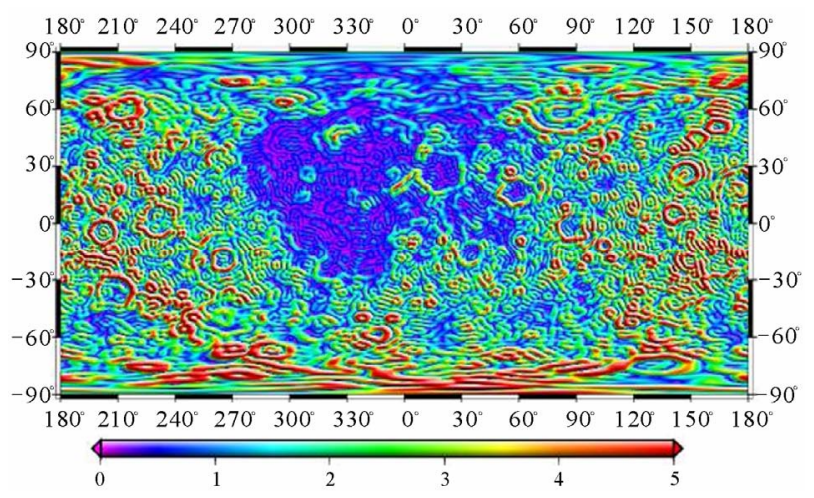

Figure 4. Gradients of lunar terrain, the X-coordinate represents longitude and the Y-coordinate represents latitude.

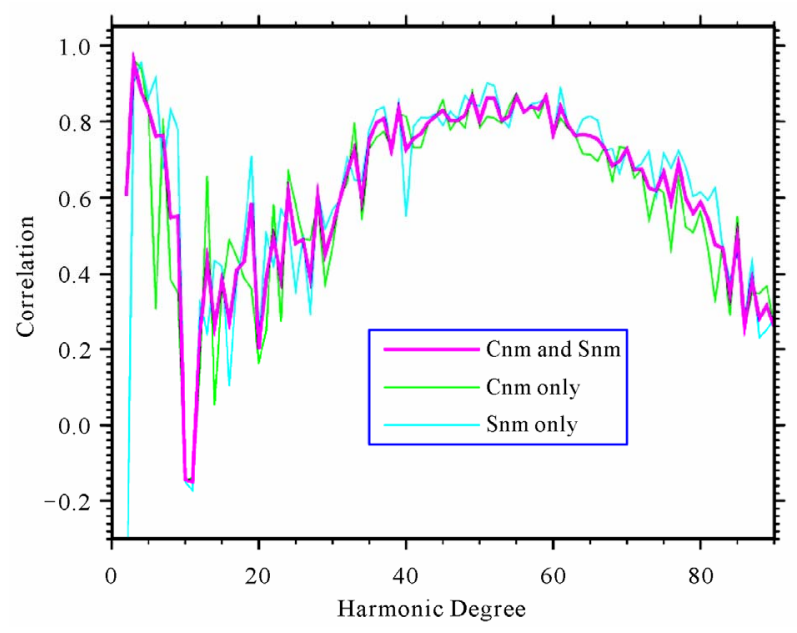

Figure 5. Correlation of SGM90d and CLTM-s01 to degree and order of 90 .

mantle uplifting. Relatively large basins more likely appear at the nearside of the Moon than the farside may be caused by the inconsistency of lunar basalt activity rather than the impact [18]. 


\section{ACKNOWLEDGEMENTS}

This study is supported in part by the National Natural Science Foundation of China under grant No. 40974016 and 40974004, the Key Laboratory of Surveying and Mapping Technology on Island and Reef of SBSM, China under grant No. 2009A02, the Research \& Innovation Team Support Program of SDUST, China, and the Science \& Technology Development Program of SBSM, China.

\section{REFERENCES}

[1] Ouyang, Z.Y. (2004) International lunar exploration progress and Chinese lunar exploration (in Chinese). Geological Science and Technology Information, 23, 1-5.

[2] Chen, S.M., Li, S.S., Mi, J., et al. (2008) Analysis and estimation on quality and reliability of lunar gravity models (in Chinese). Journal of Geomatics Science and Technology, 25, 153-156.

[3] Chen, J.Y., Ning, J.S., Zhang, C.Y., et al. (2005) On the determination of lunar gravity field in the Chinese first lunar prospector mission (in Chinese). Chinese Journal of Geophysics, 48, 275-281.

[4] Hanada, H., Iwata, T., Namiki, N., et al. (2008) VLBI for better gravimetry in SELENE. Advances in Space Research, 42, 341-346. doi:10.1016/j.asr.2007.11.003

[5] Konopliv, A.S., Asmar, A.W., Carranza, E., et al. (2001) Recent gravity models as a result of the lunar prospector mission. Icarus, 150, 1-18.

doi:10.1006/icar.2000.6573

[6] Sasaki, S., Iijima, Y., Tanaka, K., et al. (2003) The SELENE mission: Goals and status. Advances in Space Research, 31, 2335-2340. doi:10.1016/S0273-1177(03)00543-X

[7] Tanaka, S., Shiraishi, H., Kato, M., et al. (2008) The science objectives of the SELENE-II mission as the post SELENE mission. Advances in Space Research, 42, 394-401. doi:10.1016/j.asr.2007.07.002

[8] Yan, J., Ping, J., Matsumoto, K., et al. (2008) The simu- lation of lunar gravity field recovery from D-VLBI of Chang'E-1 and SELENE lunar orbiters. Advances in Space Research, 42, 337-340. doi:10.1016/j.asr.2007.11.011

[9] Kovopliv, A.S., Binder, A.B., Hood, L.L., et al. (1998) Improved gravity field of the moon from lunar prospector. Science, 281, 1476-1480.

doi:10.1126/science.281.5382.1476

[10] Konopliv, A.S. and Yuan, D.N. (1999) Lunar prospector 100th degree gravity model development. Proceedings of 30th Annual Lunar and Planetary Science Conference, Houston, 15-29 March 1999.

[11] Ping, J.S., Kono, Y., Kawano, N., et al. (2001) SELENE mission: mathematical model for SST Doppler measurements (in Chinese). Progress in Astronomy, 19, 354-364.

[12] Kato, M., Sasaki, S., Tanaka, K., et al. (2008) The Japanese lunar mission SELENE: Science goals and present status. Advances in Space Research, 42, 294-300. doi:10.1016/j.asr.2007.03.049

[13] Namiki, N., Iwata, T., Matsumoto, K., et al. (2009) Farside gravity field of the moon from four-way doppler measurements of SELENE (Kaguya). Science, 323, 900905. doi:10.1126/science. 1168029

[14] Hu, M.C. (2003) The theory and the application of contemporary geodesy (in Chinese). Surveying and Mapping Press, Beijing.

[15] Jekeli, C. (1999) An analysis of vertical deflections derived from high-degree spherical harmonic models. Journal of Geod, 73, 10-22. doi:10.1007/s001900050213

[16] Wessel, P. and Smith, W.H.F. (1995) New version of the generic mapping tools released. Eos, Transactions, American Geophysical Union, 76, 329. doi:10.1029/95EO00198

[17] Ping, J.S., Huang, Q., Yan, J.G., et al. (2009) Lunar topographic model CLTM-s01 from Chang'E-1 laser altimeter. Science in China Series G: Physics, Mechanics \& Astronomy, 52, 1105-1114.

[18] Melosh, H.J. (1989) Impact cratering: A geologic process. Oxford University Press, Oxford. 